\title{
EDITORIAL
}

\section{Networking for infectious disease}

\author{
Michael Head and colleagues describe how the Infectious Disease Research Network can \\ help microbiologists in the United Kingdom to collaborate more effectively.
}

Michael Head, Andrew Hayward and Anne Johnson are at the Department of Primary Care \& Population Sciences, University College London, Royal Free Campus, Rowland Hill Street, London, NW3 2PF UK. Correspondence to M.H. e-mail: mhead@idrn.org
Collaboration is in fashion - the Cooksey Review ${ }^{1}$ mentions it 46 times and the United Kingdom Clinical Research Collaboration incorporates the word into its moniker. Now, microbiologists in the United Kingdom can collaborate by using the Infectious Disease Research Network (IDRN), which promotes multi-disciplinary collaborations, provides information on funding opportunities, organizes training events and acts as a forum for encouraging high-quality infectious-disease research.

Over the past few years, it has been widely noted that microbiology in the United Kingdom is in dire need of increased funding and support. The Royal College of Pathologists ${ }^{2}$ suggested in 2001 that "the erosion of microbiology" is a situation in urgent need of correction, whereas in the same year the Academy of Medical Sciences ${ }^{3}$ concluded that most medical-microbiology departments in the United Kingdom are "in a state of torpor." They acknowledged that advances had been made in diagnostic and molecular techniques, but highlighted the need for closer engagement between basic scientists, clinicians, epidemiologists and healthprotection specialists. Interdisciplinary relationships were also recommended in Fighting Infection ${ }^{4}$, the House of Lords report.

The IDRN, which is free to join, was formed in 2001, and is funded by the National Coordinating Centre for Research Capacity Development. It was initially piloted as a London network, but now engages with researchers across the United Kingdom. A national consultation exercise $^{5}$ that was carried out by the IDRN in 2005 received around 200 responses. This exercise revealed a previously invisible demand for an infrastructure that can provide advice on governance and support for the epidemiological, clinical and statistical aspects of study design. The IDRN has more than 1,100 members, with microbiologists being well represented among epidemiologists, immunologists, virologists, clinicians and statisticians.

One of the main outreach activities of the IDRN is

After workshops, the IDRN organizes more-focused protocol-development groups that concentrate on specific research questions, which brings disciplines together with the aim of submitting a grant application to the appropriate funder. The IDRN provides administrative support at these meetings, which includes carrying out literature searches for grant proposals. Appropriate collaborators can also be found to lend advice on study design. The IDRN has been involved in the development of the Food Standards Agency National Intestinal Infectious Disease Study and the Medical Research Council Flu-Watch cohort. We have also provided support for successful fellowship applications. A detailed list of research outcomes and publications is available at http://www.idrn.org/research.php.

We run training workshops on topics such as 'grantsmanship', molecular microbiology, the Human Tissue Act and the EU Clinical Trials Directive. Increasingly, we interface with funding organizations: we organized a Challenge workshop with the United Kingdom Clinical Research Collaboration (UKCRC) on research priorities in health-care-associated infections and antimicrobial resistance and helped to launch the UKCRC Partners Translational Infection Research Initiative.

Among our most widely used resources are IDRN bulletins, which contain up-to-date information on available funding and training opportunities. Our online searchable database of researchers enables colleagues to link up across disciplines.

The IDRN provides a model of how a small administrative infrastructure, with academic input from members, can initiate and support a range of collaborations, training events and information resources, thereby ultimately leading to better collaborative research, and we encourage UK researchers to join us now.

\footnotetext{
. Cooksey, D. A review of UK health research funding (Her Majesty's Stationery Office, London, 2006)

2. The Royal College of Pathologists. Consultant workload and staffing in medical microbiology and virology. Report of a working group of The Royal College of Pathologists [online] http://www.rcpath.org/resources/ pdf/C014-ConsultantWorkloadStaffingMedMicro-Apr01.pdf (2001).
} workshops, which aim to identify research priorities and foster collaborations. Workshops on antimicrobial resistance, tuberculosis, hepatitis $\mathrm{C}$, bioterrorism, prison health, food safety, sexual health and health protection have all been held to date. Planned topics for 2008 include lymphocyte kinetics, systemic fungal infections and outbreak investigations for research and zoonoses. 